\title{
Coarse-grain simulation of viscous flow and stamp deformation in nanoimprint
}

\author{
V. Sirotkin, A. Svintsov, and S. Zaitsev ${ }^{\text {a) }}$ \\ Institute of Microelectronics Technology, RAS, Chernogolovka, Moscow 142432, Russia \\ H. Schift \\ Laboratory for Micro- and Nanotechnology, Paul Scherrer Institute, 5232 Villigen PSI, Switzerland
}

(Received 9 June 2007; accepted 23 October 2007; published 10 December 2007)

\begin{abstract}
A refined coarse-grain software for the simulation of thermal nanoimprint lithography is described. This software takes into account the stamp bending during squeeze flow and is able to provide results well suited for the quantitative prediction of the residual resist thickness with an accuracy better than 10\%. () 2007 American Vacuum Society. [DOI: 10.1116/1.2812534]
\end{abstract}

\section{INTRODUCTION}

The design of "an optimum" stamp geometry is an essential issue in thermal nanoimprint lithography (NIL) (see Refs. 1 and 2 for example). This is easy for dense arrangements of stamp patterns such as large areas gratings, but not for applications where large and small features have to be integrated in one design, e.g., for sensors with fluidic and optical elements. Because of the high pressure needed for thermal NIL, the printing setup is able to comply, which enables the stamp to conform to stamp pattern variations. Design optimization is often an easy way to improve the homogeneity simply by rearrangement of patterns or adding of auxiliary structures. This optimization must exclude extreme distributions of cavities and protrusions with large local variations of protrusion densities and sizes. In the latter case, the resist has to flow laterally over large distances, which results in a large residual layer thickness. These areas are characterized by a locally increased value of pressure, which results in a nonuniform deformation of the stamp and substrate and, consequently, in inhomogeneities of the residual layer thickness. If they fall outside of specific tolerances, then the execution of further processing, e.g., pattern transfer, will be difficult.

The greatest benefit of the implementation of simulation software can be achieved if stamp optimization is performed before expensive stamp manufacturing begins. To do this, an effective simulation tool is required for the prediction of the residual layer thickness from a given stamp structure, not only locally but over large stamp surfaces. Then, stamp bending has to be taken into account, which is typical is squeeze flow of viscous resists and deals with lateral dimensions of a few micrometers up to millimeters.

Modeling of the resist viscous flow at the detail level (for a single cavity) is widely covered in the literature (see Ref. 3 and references therein). However, approaches for detailed (quantitative) analysis of resist spreading in large areas are not described. In this article, a software for simulation of NIL at the structure-scale level is presented, which is based on a refined version of the coarse-grain method. In contrast

${ }^{a)}$ Electronic mail: zaitsev@iptm.ru to the previous method, ${ }^{4,5}$ the current version is able to integrate bending effects, which allows for the determination of the residual layer thickness within a precision of $10 \%$.

\section{MATHEMATICAL MODEL}

In Ref. 5, the mathematical model for the simultaneous calculation of the resist viscous flow in NIL and the stamp/ substrate deformation has been introduced. The model specifies the two-dimensional temporal distributions of the pressure and the normal displacement of the stamp/substrate surface. The model has the following input parameters: the distribution of the stamp relief height $h(x, y)$, the initial resist thickness $d_{0}$, the stamp velocity $V_{\text {st }}$, the total force $F$ acting on the stamp, the duration of the imprinting process $T$, and the dynamic viscosity of the resist $\eta$. In the model, at every point in time $t$, the pressure distribution $P(x, y, t)$ is calculated from the following problem:

$$
\begin{aligned}
& \nabla\left\{[D(x, y, t)+h(x, y)]^{3} \nabla P(x, y, t)\right\} \\
& =12 \eta \frac{\partial D(x, y, t)}{\partial t}, \quad(x, y) \in \Omega_{f}, \quad t \in(0, T], \\
& P(x, y)=0, \quad(x, y) \in \bar{\Omega} / \Omega_{f}, \\
& D(x, y, t)=d_{0}-\int_{0}^{t} V_{\mathrm{st}}(\mathrm{s}) d s+\delta_{\mathrm{st}}(x, y, t)+\delta_{\mathrm{sb}}(x, y, t),
\end{aligned}
$$

where $\Omega$ is the considered domain of the stamp, $\Omega_{f}$ is the part of $\Omega$, in which all cavities are filled with the resist, and $D(x, y, t)$ is the temporal distribution of the residual layer thickness. In Eq. (1), the zero value of the pressure on the boundary of the considered domain $\Omega$ and in the unfilled cavities corresponds to the imprinting process performed in vacuum.

Note that Eq. (1) is derived from three-dimensional Navier-Stokes equations with the understanding that the resist has very high viscosity and its motion is largely directed along the substrate surface.

For the calculation of the elastic normal displacements $\delta_{\mathrm{st}}$ and $\delta_{\mathrm{sb}}$, the stamp and the substrate are represented as semi- 
infinite regions (an elastic medium bounded by a plane). In this situation, the displacements are described by the following expression:

$$
\begin{gathered}
\delta(x, y, t)=\frac{1-\sigma^{2}}{\pi E} \iint_{\Omega} \frac{P\left(x^{\prime}, y^{\prime}, t\right) d x^{\prime} d y^{\prime}}{\left(x-x^{\prime}\right)^{2}+\left(y-y^{\prime}\right)^{2}}, \\
(x, y) \in \Omega, \quad t \in(0, T],
\end{gathered}
$$

where $\sigma$ is Poisson's ratio and $E$ is modulus of elasticity. ${ }^{6}$

It must be emphasized that a comparison of simulated and experimental results presented in Sec. IV demonstrates the validity of the proposed deformation model. However, if required, the displacements $\delta_{\mathrm{st}}$ and $\delta_{\mathrm{sb}}$ can be specified using alternative models of the stamp and substrate deformations.

The model allows the calculation of the pressure distribution under several loading regimes: constant imprint velocity or constant applied force. Note that the solution of Eq. (1) is fully conditioned by the stamp velocity. If the imprinting process is realized under a fixed force $F^{*}$, in which case no prior knowledge of the value of the stamp velocity is available, then the required pressure distribution is obtained from Eqs. (1) and (2) at the following additional condition:

$$
F^{*}=\iint_{(x, y) \in \Omega_{f}} P(x, y, t) d x d y, \quad t \in\left(t^{*}, T\right] .
$$

In the simulation, the following implementation of the imprint process is analyzed: the stamp moves with the constant velocity so long as the total force acting on the stamp $F$ $<F^{*}$; then the process is realized under the fixed force $F^{*}$.

\section{COARSE-GRAIN METHOD}

By the numerical approximation of the above-described mathematical model, a special finite difference method is applied. The method provides a high precision of simulation results by using a reasonably coarse grid. ${ }^{4,5}$ Here, a refined version of this (coarse-grain) method is presented.

Suppose that the considered domain is a rectangle $\Omega$ $=\left(0, L^{x}\right) \times\left(0, L^{y}\right)$. A uniform grid is introduced on $\Omega$ :

$$
\begin{array}{ll}
x_{i}=i h^{x}, & i=\overline{\left(0, N^{x}\right)}, \quad h^{x}=L^{x} / N^{x} ; \\
y_{j}=j h^{y}, \quad j=\overline{\left(0, N^{y}\right)}, \quad h^{y}=L^{y} / N^{y} ;
\end{array}
$$

together with the grid functions

$$
\begin{aligned}
& P_{i, j}=P\left(x_{i}, y_{i}\right) \text { and } D_{i, j}=D\left(x_{i}, y_{i}\right), \\
& (i, j) \in \bar{\omega}=\overline{\left(0, N^{x}\right)} \times \overline{\left(0, N^{y}\right)} .
\end{aligned}
$$

The domain $\Omega$ is mapped by rectangular cells so that the mesh points are the centers of the cells and the edges of the cell intersect the mesh lines at midpoints. Then, finite difference approximation of Eq. (1) is derived as follows:

$$
\begin{aligned}
& \frac{\phi_{i, j}}{h^{x} h^{y}} \Lambda(t) P_{i, j}(t)-\frac{1-\phi_{i, j}}{\beta_{i, j}^{2}} D_{i, j}^{3}(t) P_{i, j}(t) \\
& \quad=12 \eta \phi_{i, j}^{2} \frac{D_{i, j}(t)-D_{i, j}(t-\tau)}{\tau}, \quad(i, j) \in \omega, \quad t \in(0, T],
\end{aligned}
$$

$$
P_{i, j}=0, \quad(i, j) \in \bar{\omega} / \omega,
$$

where $\tau$ is the nonuniform step of time discretization, $\phi_{i, j}$ is the ratio of the protrusion area to the total area of the $(i, j)$ cell, and $\beta_{i, j}$ is the characteristic size of protrusions in the $(i, j)$ cell. In Eq. (3), the following notations are introduced:

$$
\begin{aligned}
\Lambda P_{i, j}= & \Lambda^{x} P_{i, j}+\Lambda^{y} P_{i, j}, \\
\Lambda^{x} P_{i, j} & =K_{i, j}^{x}\left(P_{i+1, j}-P_{i, j}\right)-K_{i-1, j}^{x}\left(P_{i, j}-P_{i-1, j}\right), \\
\Lambda^{y} P_{i, j}= & K_{i, j}^{y}\left(P_{i, j+1}-P_{i, j}\right)-K_{i, j-1}^{y}\left(P_{i, j}-P_{i, j-1}\right), \\
K_{i, j}^{x}= & \left\{\int _ { x _ { i } } ^ { x _ { i + 1 } } \left[\int _ { y _ { j } - h ^ { y } / 2 } ^ { y _ { j } + h ^ { y } / 2 } \left[\frac{1}{2}\left(D_{i, j}+D_{i+1, j}\right)\right.\right.\right. \\
& \left.\left.+h(x, y)]^{3} d y\right]^{-1} d x\right\}, \\
K_{i, j}^{y}= & \left\{\int _ { y _ { j } } ^ { y _ { j + 1 } } \left[\int _ { x _ { i } - h ^ { x } / 2 } ^ { x _ { i } + h ^ { x } / 2 } \left[\frac{1}{2}\left(D_{i, j}+D_{i, j+1}\right)\right.\right.\right. \\
& \left.\left.+h(x, y)]^{3} d x\right]^{-1} d y\right\} .
\end{aligned}
$$

The right-hand side of Eq. (3) specifies the resist flow in the $(i, j)$ cell caused by stamp motion. The first term in the lefthand side of Eq. (3) determines exchange of resist between the $(i, j)$ cell and adjacent cells. The second term describes the resist spreading inside the $(i, j)$ cell.

The refined coarse-grain method based on the finite difference problem [Eq. (3)] allows obtaining more realistic simulation results. Moreover, for the numerical solution of Eq. (3), multigrid approach can be efficiently applied. This opens the way to the coarse-grain simulation of NIL for the full area of 4-6 in. stamps.

\section{EXPERIMENT VERSUS COARSE-GRAIN SIMULATION}

The refined coarse-grain method has been realized in software for the verification of stamp structures on standard personal computers by using the Graphic Data System (GDS) data of the stamp design. (Graphic Data System is a database file format for integrated circuit layout data exchange).

Here, the software is implemented for the simulation of an imprint process using a structure from the experimental test stamp of the $\mathrm{NaPa}$ project. ${ }^{7}$ The structure measures 2 $\times 2 \mathrm{~mm}^{2}$ and contains protrusions of varying width and coverage (see Fig. 1). Imprinted samples of this structure demonstrate inhomogeneities of the residual layer thickness related to the nonuniform deformation of stamp and substrate as well as to incompletely molded cavities. Two variants of the test structure are analyzed: with direct [see Fig. 1(a)] and inverse [see Fig. 1(c)] relief.

Figures 2-7 give calculation results obtained for the test structure. By modeling, a $128 \times 128$ pixel grid is applied. Simulation parameters are chosen as follows: the stamp cavities are $200 \mathrm{~nm}$ in depth, the initial resist thickness $d_{0}$ is 

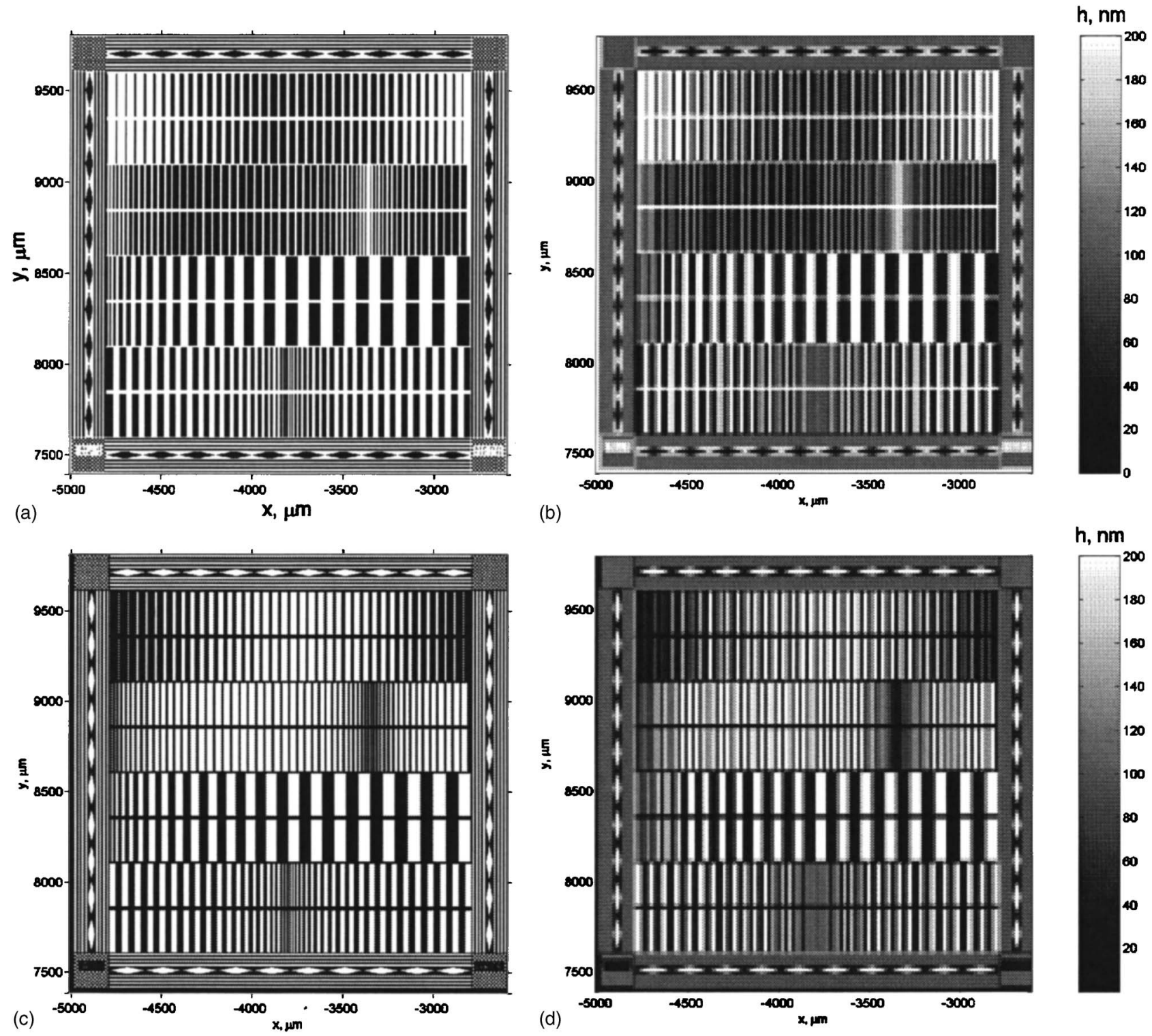

(b)

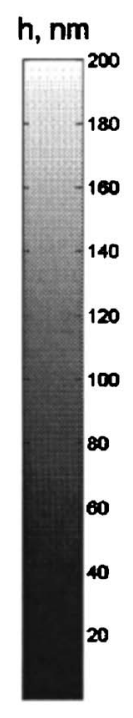

(d)

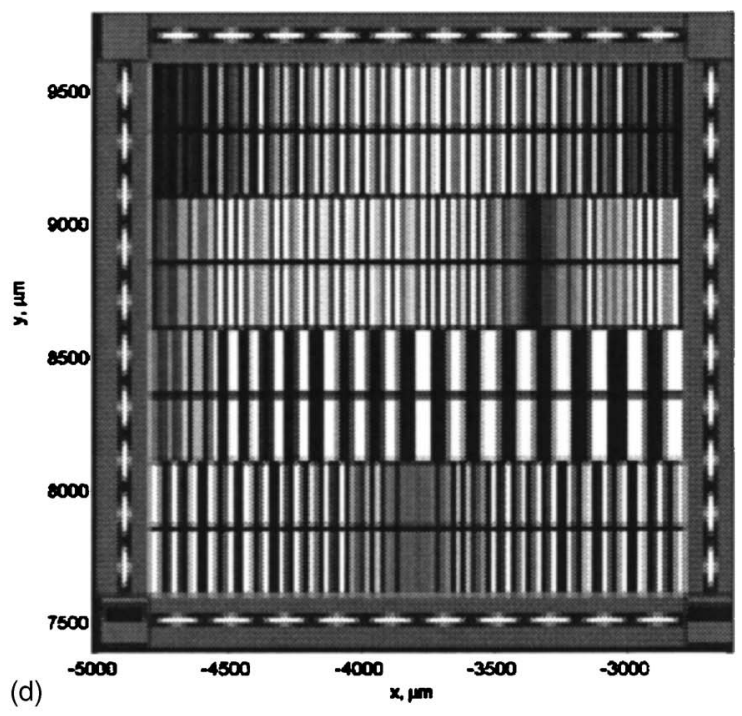

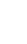

FIG. 1. Original relief $h(x, y)$ of the test structure (cavities and protrusions are painted white and black, respectively): (a) with direct relief and (c) with inverse relief. [(b) and (d)] Presentation of the same structures by the coarse-grain simulation on $128 \times 128$ pixel grid.

$300 \mathrm{~nm}$, the dynamic viscosity of the resist $\eta$ is equal to $10^{4} \mathrm{~Pa} \mathrm{~s}$, modulus of elasticity $E$ is $10^{11} \mathrm{~Pa}$, and Poisson's ratio $\sigma$ is equal to 0.2 . The stamp moves with the constant velocity $V_{\mathrm{st}}=1 \mathrm{~nm} / \mathrm{s}$ until the total force acting on the stamp reaches $F^{*}=20 \mathrm{~N}$. The imprinting process lasts $200 \mathrm{~s}$.

The main output information of the imprint process simulation is the distribution of the resist thickness $H(x, y)$ (see Figs. 2 and 4), which can be specified as follows:

$$
H(x, y)=D(x, y, T)+h(x, y)-H_{e}(x, y, T),
$$

where $D$ is the residual layer thickness, $h$ is the stamp relief height, and $H_{e}$ is the underfilling height of the stamp cavities.

Note that the coarse-grain simulation applies an approximation of the stamp relief height $h$. This approximation can be quite rough (see Fig. 1). If by calculating the distribution of the resist thickness $H$ the "rough" approximation of $h$ is used, then the obtained result is unsuitable for interpretation. Applying in Eq. (4) a closer approximation of $h$ (for which a finer pixel grid is used), this problem can be overcome. It should be emphasized that the described trick is absolutely allowable because it is related exclusively to visualization but not to any recalculation of simulation results.

In Figs. 3 and 5, simulated and experimental distributions of the resist thickness are compared for the test structure with direct and inverse relief, respectively. The experimental results are obtained by profilometer measurements of the replicas after imprint of the considered structures into resist. For better visualization of the comparison, Fig. 6 shows the same results recalculated in the distributions of the residual layer thickness $D$ [see Eq. (4)]. It should be noted that the experimental and simulated results agree very closely (maximum difference is less than $10 \%$ ). 


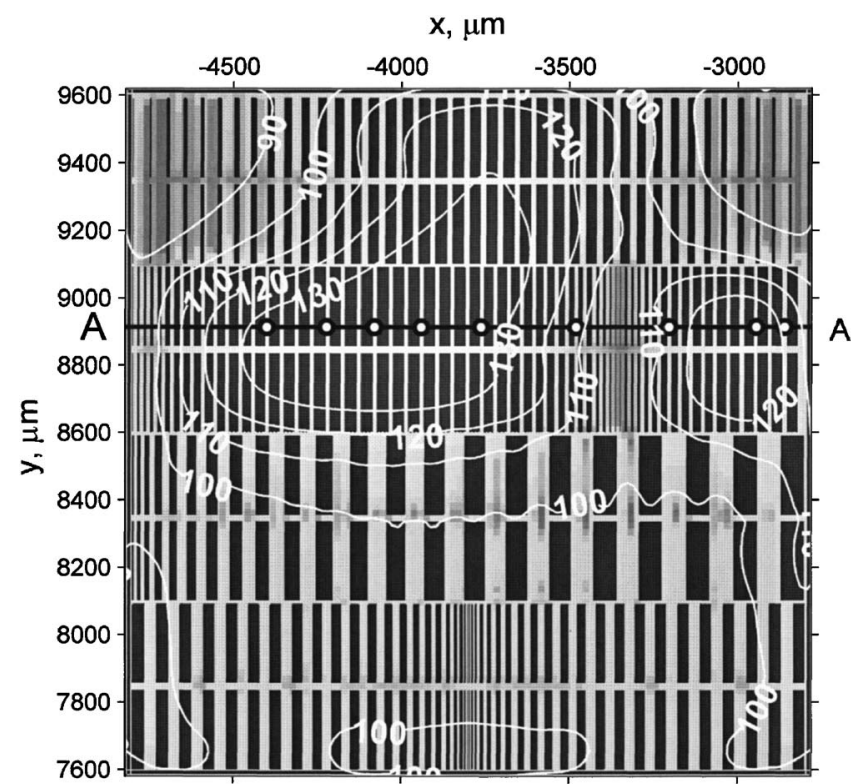

$\mathrm{H}, \mathrm{nm}$

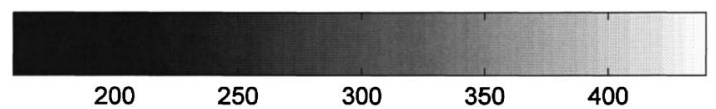

Fig. 2. Calculated distribution of the resist thickness $H(x, y)$ for the test structure with direct relief. For the "fine" visualization of $H(x, y)$, the approximation of the stamp relief height $h(x, y)$ on a $1024 \times 1024$ pixel grid is applied. White isolines indicate the distribution of the stamp and substrate deformation (numbers indicate the elastic displacement in nanometers). The horizontal line (A-A) and the circles $(\bigcirc)$ mark a cross section and points where simulated and measured values of the resist thickness are compared (see Fig. 3).

In closing, as an illustration, Fig. 7 compares the considered imprint process with the process fulfilled at $F^{*}=10 \mathrm{~N}$. The processes are analyzed in the case of the test structure

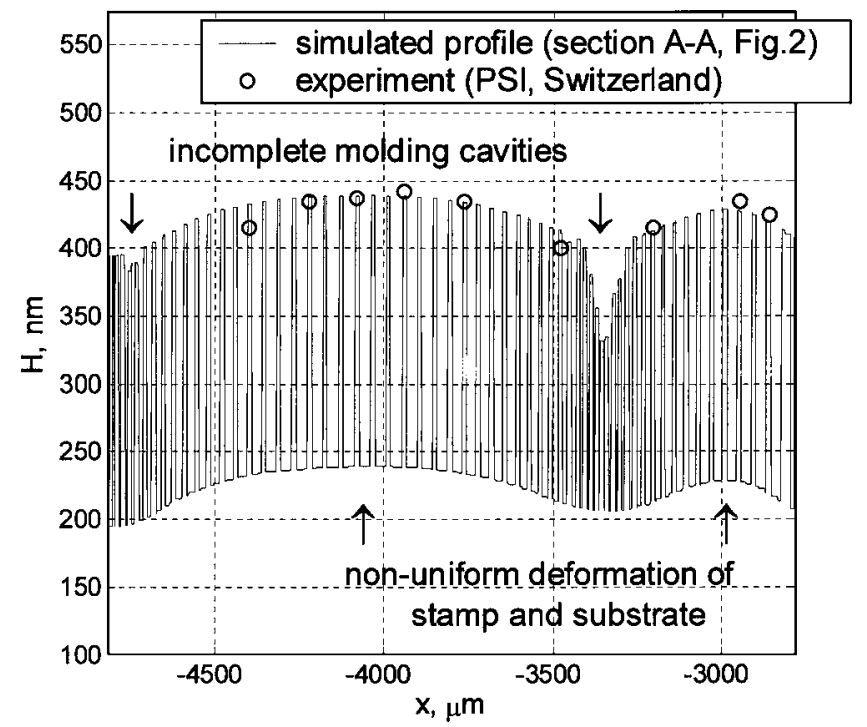

FIG. 3. Comparison of measured and simulated values of the resist thickness $H(x, y)$ for the test structure with direct relief.

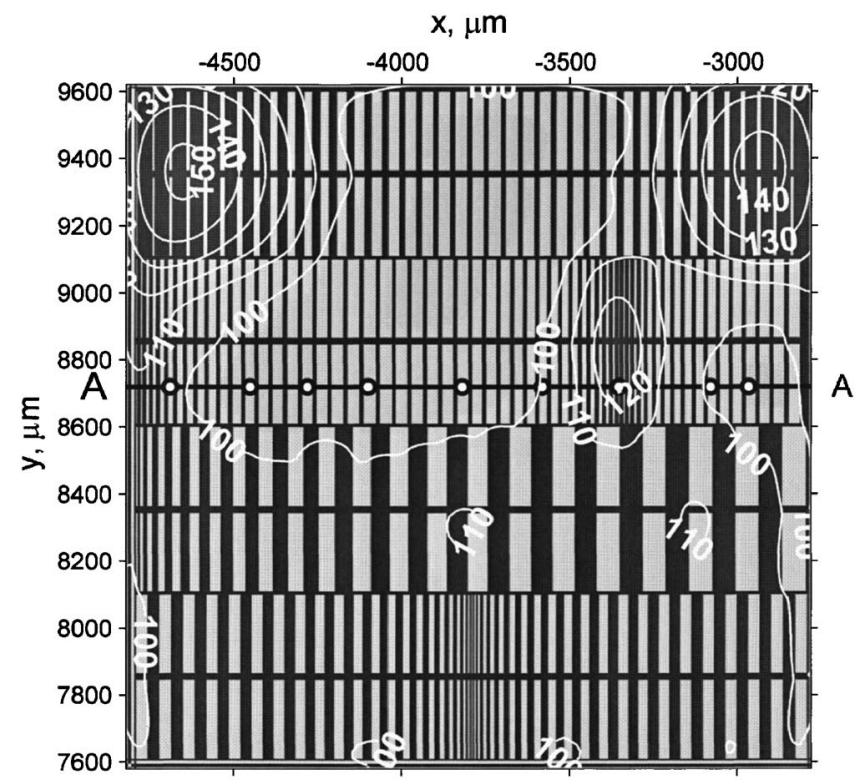

$\mathrm{H}, \mathrm{nm}$

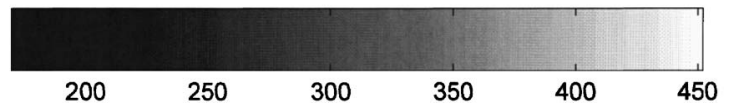

FIG. 4. Calculated distribution of the resist thickness $H(x, y)$ for the test structure with inverse relief. White isolines indicate the distribution of the stamp and substrate deformation (numbers indicate the elastic displacement in nanometers). The horizontal line (A-A) and the circles $(\mathrm{O})$ mark a cross section and points where simulated and measured values of the resist thickness are compared (see Fig. 5).

with direct relief. As indicated in the picture, once the total force acting on the stamp has reached $F^{*}$, a sharp decrease of the stamp velocity is observed.

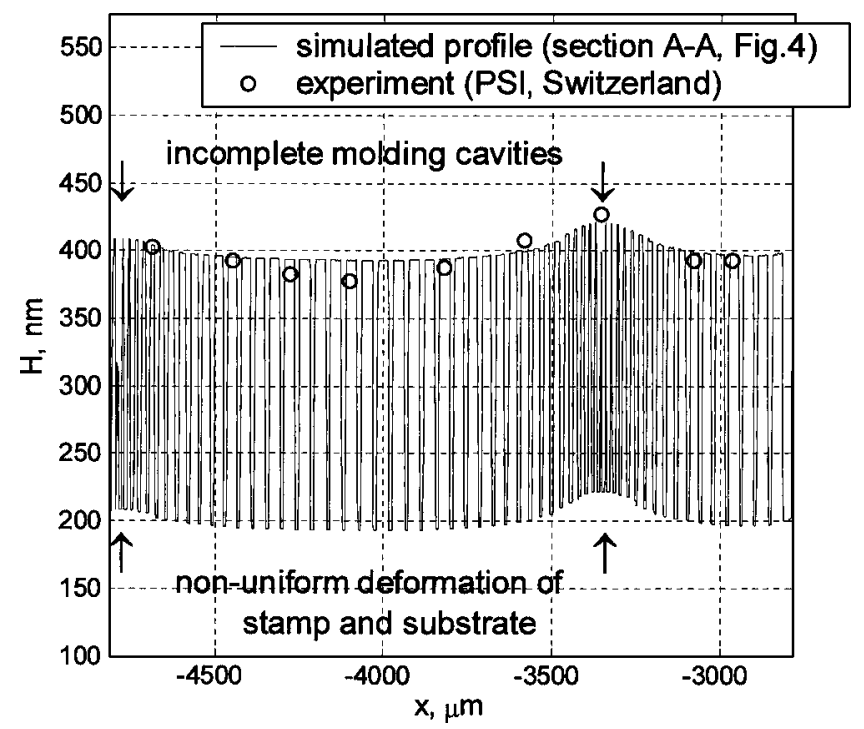

FIG. 5. Comparison of measured and simulated values of the resist thickness $H(x, y)$ for the test structure with inverse relief. 


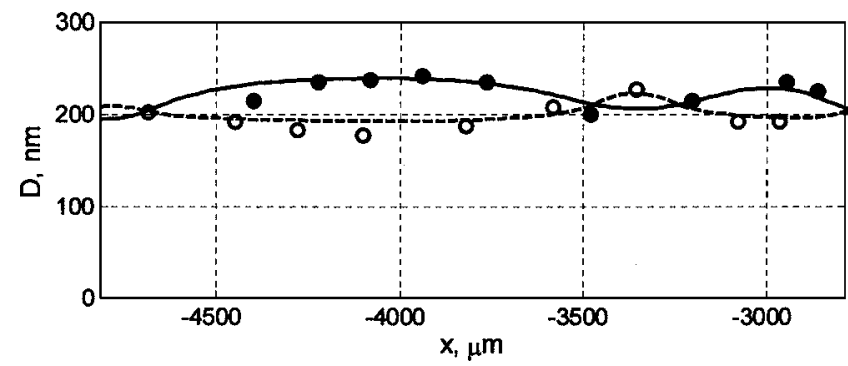

FIG. 6. Comparison of measured $(\bullet$, direct relief $\bigcirc$, inverse relief) and simulated (solid line, direct relief; dashed line, inverse relief) values of the residual layer thickness $D(x, y)$ for the test structure (sections A-A on Figs. 2 and 4). The simulation confirms the high residual layer thickness variation ranges (from 195 to $240 \mathrm{~nm}$ for direct relief and from 180 to $225 \mathrm{~nm}$ for inverse relief) with a precision of $10 \%$, which is due to the variation of the ability of polymer to flow.

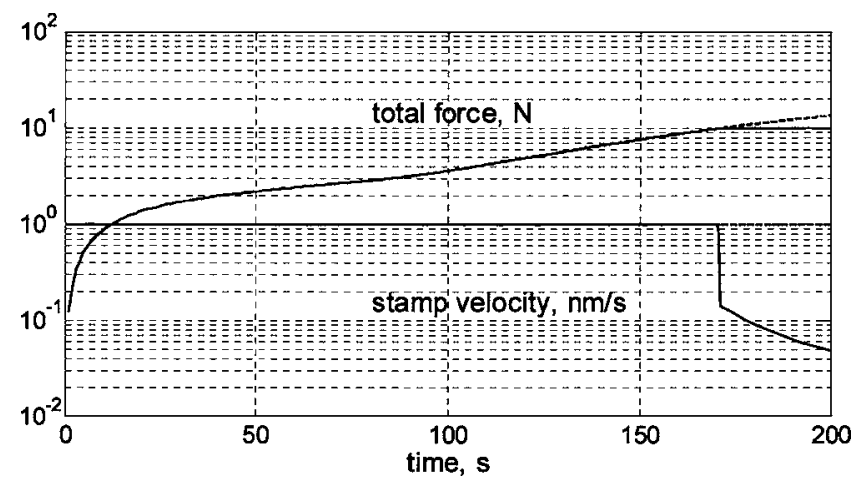

FIG. 7. Test structure with direct relief. Time variation of the total force and the stamp velocity for two imprinting process distinct in $F^{*}$ (maximum allowed values of total force acting on the structure): $20 \mathrm{~N}$ (dashed lines) and $10 \mathrm{~N}$ (solid lines). The first process stops short of $F^{*}$. In the second process, the total force has reached $F^{*}$. At this moment, changeover from regime with constant velocity $(1 \mathrm{~nm} / \mathrm{s})$ to regime with fixed force (see Sec. II for details) is observed.

\section{CONCLUSION}

The refined coarse-grain method for simultaneous calculation of the viscous flow of resist and the stamp and substrate deformation has been developed. The method has been realized in software for the verification of stamp structures. The comparison of experimental and simulated results shows that the coarse-grain modeling allows for predicting the residual layer thickness with accuracy better than $10 \%$. Moreover, the presented software demonstrates a high computational performance. Typical simulation times for the test structure with $2 \times 2 \mathrm{~mm}^{2}$ area are less than $20 \mathrm{~min}$ on an AMD Athlon 64, $2400 \mathrm{MHz}$ processor. We expect that the method can be easily extended to large area stamps and process parameters used for typical NIL applications.

\section{ACKNOWLEDGMENT}

The partial support of the EC-funded project $\mathrm{NaPa}$ (Contract No. NMP4-CT-2003-500120) is gratefully acknowledged.

${ }^{1}$ H. Schift and L. J. Heyderman, Alternative Lithography, edited by C. M. Sotomayor Torres, Nanostructure Science and Technology (Kluwer Academic Dordrecht/Plenum, New York, 2003), Chap. 4, pp. 47-76.

${ }^{2}$ L. J. Guo, J. Phys. D 37, R123 (2004).

${ }^{3}$ H. D. Rowland, A. C. Sun, P. R. Schunk, and W. P. King, J. Micromech. Microeng. 15, 2414 (2005).

${ }^{4}$ V. Sirotkin, A. Svintsov, S. Zaitsev, and H. Schift, Microelectron. Eng. 83, 880 (2006)

${ }^{5}$ V. Sirotkin, A. Svintsov, H. Schift, and S. Zaitsev, Microelectron. Eng. 84, 868 (2007).

${ }^{6}$ L. D. Landau and E. M. Lifshitz, Theory of Elasticity (Pergamon, Oxford, 1986), Vol. 7, p. 108

${ }^{7} \mathrm{NaPa}$ project, Integrated Project, EU Sixth Frame Program (http:// www.phantomsnet.net/NAPA/index.php). 